Marcus Rohnke · Timo Best · Jürgen Janek

\title{
Controlled electrochemical growth of silver microwires
}

\begin{abstract}
The cathodic deposition of silver whiskers as microwires in the electrochemical cell $\mathrm{Ag} / \mathrm{AgBr} / \mathrm{Ag}$ has been investigated. From growth experiments observed with an optical microscope, we conclude that the growth process occurs exclusively at the interface of the silver wires with the solid electrolyte. The basal contacts of the microwires show different morphologies without clear correlation to the substrate. The silver crystals often show a hexagonal morphology, but XRD gives no definite information on the structure of the wires. The measured exchange current density is in the order of $100 \mathrm{~mA} / \mathrm{cm}^{2}$. The growth rate and the diameter of the microwires can be controlled by variation of the current.
\end{abstract}

Keywords Whisker Cathodic deposition · Solid electrolyte $\cdot$ Microstructure $\cdot$ Interface

\section{Introduction}

Electrochemical micro- and nano-structuring is currently of strong interest in various fields of application, primarily due to the expected low costs. In this context first attempts to apply solid state electrochemical techniques have recently been reported by Spangenberg et al. [1] using microelectrodes and by Terabe et al. [2] using scanning tunneling microscope (STM). It is the purpose of this paper to report on first attempts to apply the electrochemically controlled whisker growth on solid electrolytes as a route towards the preparation of metal microwires and microwire arrays.

Whisker growth by supersaturation of mixed ionic/ electronic conductors like $\mathrm{Ag}_{2} \mathrm{~S}$ [3] and $\mathrm{Ag}_{2} \mathrm{Se}$ [4] or by

M. Rohnke $\cdot$ T. Best $\cdot$ J. Janek $(\bowtie)$

Physikalisch-Chemisches Institut,

Justus-Liebig-Universität Gießen,

Heinrich-Buff-Ring 58, 35392 Gießen, Germany

E-mail: juergen.janek@phys.chemie.uni-giessen.de

Tel.: + 49-641-9934500

Fax: + 49-641-9934509 chemical reduction of silver halides [5] has early been reported, but suffers from a lack in reproducibility of the product morphology. The cathodic deposition of whisker-type deposits on single crystalline solid electrolyte structures appears to be a reasonable basis for a much more refined product morphology, being well adjustable by the experimental parameters.

Electrochemical deposition and dissolution of metals in liquid electrolytes are well documented and investigated as classical examples for electrode processes and as important elements of many technological processes (e.g. galvanization, battery charging or discharging) [6]. The low viscosity of liquid electrolytes and continuous homogenization by convection typically leads to wellknown dendrite- or fractal-like growth morphologies of the cathodic deposits, if no surface active compounds are added. Both the corresponding anodic and cathodic phenomena in solid electrolytes are by far less investigated. In particular, the cathodic deposition of metals on solid electrolytes is not well understood, as Spangenberg et al. [1] pointed out. Mechanical effects, related to the elastic and plastic deformation of the solid phases, and thus, to the presence of non-equilibrium defects (mainly dislocations) play a decisive role for metal dissolution or growth in or on a solid electrolyte [7], compared to their less significant role in liquid state processes. From this point of view, our study also adds basic knowledge to the interfacial kinetics of solid electrolytes.

\section{Materials and methods}

Materials

$\mathrm{AgBr}$ single crystals were purchased from Korth Kristalle $\mathrm{GmbH}$, cut, grinded and chemically polished with $\mathrm{Na}_{2} \mathrm{~S}_{2} \mathrm{O}_{3}$ solution $(c=2 \mathrm{~mol} / \mathrm{l})$. Silver cathodes and anodes were cut from silver foil (99.9\%, Chempur) and prepared by grinding and polishing with diamond suspension $(1 / 4 \mu \mathrm{m})$. 


\section{Cell arrangement}

The whisker growth experiments have been performed in two different experimental setups. The first setup consists of a conventional sample holder for solid state galvanic cells, which is placed into a furnace as described in [8]. The second setup is a small heating stage under an optical microscope, which we designed particularly for whisker growth experiments. In both setups the electrochemical cell can be heated up to $300{ }^{\circ} \mathrm{C}$, and mechanical pressure can be applied orthogonal to the interface $\mathrm{AgBr} / \mathrm{Ag}$. Thus, it is possible to observe the process at the anodic interface by using the optical microscope. The cell was connected to a standard potentiostat (model $263 \mathrm{~A}$, Perkin Elmer) with computer controlled data acquisition. Electric currents between 150 and $300 \mu \mathrm{A}$ have been applied to geometrical electrode areas with a size of approx. $35 \mathrm{~mm}^{2}$, corresponding to the mean current densities in the order of $1 \mathrm{~mA} / \mathrm{cm}^{2}$ (assuming the whole geometric electrode area to be electrochemically active). The corresponding electrode overvoltages during whisker growth and dissolution were in the order of a few millivolts. In Fig. 1, the experimental cell for the electrochemical growth of whiskers is depicted schematically.

Ex situ investigations of the whiskers were performed by field emission scanning electron microscopy (FESEM) and X-ray diffraction (XRD). The whisker roots were prepared by mechanical separation of the silver whiskers from the solid electrolyte and subsequent cleaning with $\mathrm{Na}_{2} \mathrm{~S}_{2} \mathrm{O}_{3}$ solution.

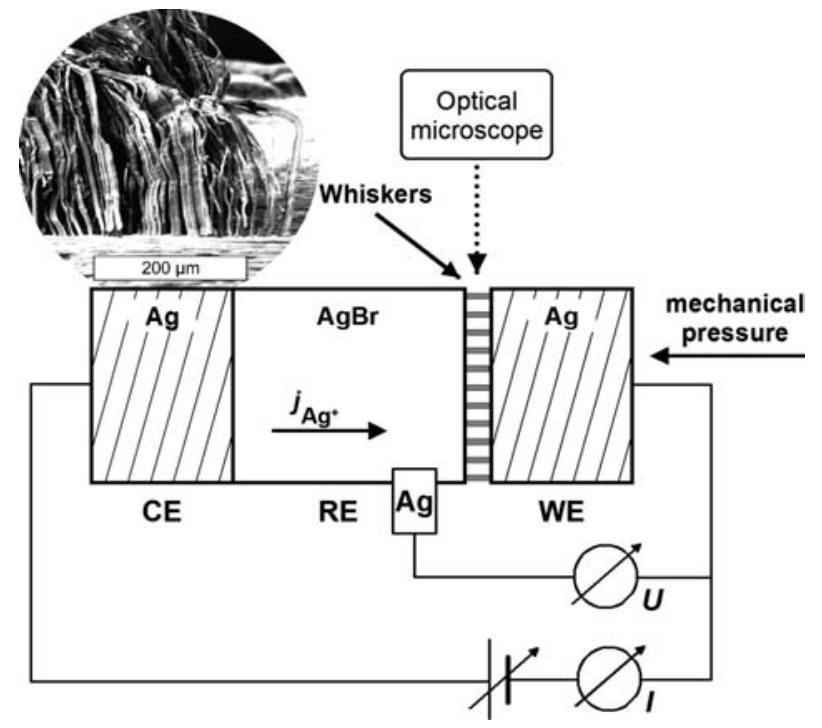

Fig. 1 Experimental setup (schematical) for the electrochemical growth of silver whiskers and SEM graph of silver whisker array grown electrochemically on $\mathrm{AgBr}$

\section{Results}

Morphology of whiskers

The typical whisker density (average over large area) is in the order of $10^{6} / \mathrm{cm}^{2}$, showing a relatively homogeneous distribution in case of a good electrode contact. If the electrode contact is restricted to a few point contacts, only dendrite formation along the electrolyte surface is observed.

Some whiskers clearly show a hexagonal morphology, see Fig. 5d, but most whiskers show a less welldefined morphology. Typical slip bands of single crystals are found and prove the high degree of crystallinity of the whiskers. The preparation of whiskers for XRD investigations is difficult, mechanical deformation of the thin metal whiskers during removal from the electrochemical cell cannot be avoided. Nevertheless, we attempted to determine the structure of single whiskers by XRD, employing an IPDS technique. So far, first results from IPDS measurements indeed indicate a hexagonal symmetry with a unit cell of the dimensions $a$ $=b=0.298 \mathrm{~nm}$ and $c \approx 0.705 \mathrm{~nm}$. However, the structure determination bears severe problems: due to the slip bands of the whiskers it is not possible to determine precisely all three unit cell constants. The cell constant $c$ in the direction of crystal growth shows a large error. Thus, the determination of the $c$ parameter is difficult, most probably due to a large number of stacking faults of the (111) layers. Twinning of whiskers can also not be excluded, and thus, future efforts will be directed towards a transmission electron microscope (TEM) characterisation. At this stage of the investigation, we conclude that the electrochemical growth of silver whiskers at $300{ }^{\circ} \mathrm{C}$ appears to lead to single, twin or multi-twin crystals with close packed silver layers in plane with the substrate surface, incorporating a high density of stacking faults.

\section{Influence of cathode geometry}

As shown in Figs. 1 and 2, the deposition takes place by the formation of a bundle of equally sized silver whiskers at a macroscopically extended plane electrode, giving the appearance of a whisker or microwire "array". It should be noted that array-like whisker growth is only observed at plane cathodes in good mechanical contact with the electrolyte. The surface preparation of the cathode has no detectable influence on the process. Thus, thin plastic electrodes which provide a good initial contact to the solid electrolyte are recommended.

\section{Influence of substrate}

Test experiments have been performed on both single crystalline $\mathrm{AgBr}$ and $\mathrm{AgCl}$ substrates. In contrast to the 
whisker growth on $\mathrm{AgBr}$, the metal is always deposited in a dendritic morphology on $\mathrm{AgCl}$, as exemplified by Spangenberg et al. [1]. Thus, we mainly used $\mathrm{AgBr}$ as the solid electrolyte substrate for whisker growth.

In addition, we performed growth experiments with the electrochemical cells $\mathrm{Cu} / \mathrm{CuBr} / \mathrm{Ag}$ and $\mathrm{Cu} / \mathrm{AgBr} / \mathrm{Ag}$. In both cases, the growth of metal whiskers is observed. In the first case, pure copper whiskers are precipitated. In the second case the whiskers consist of silver with a small amount of copper impurity $(<0.3 \%)$. Microprobe measurements show a slight increase of the copper amount towards the whisker roots. This is expected due to the fact that it takes a certain time until the copper is diffused through the solid electrolyte. Changing the anode in a controlled manner from one metal to the other one, bimetallic whiskers might be grown.

\section{Experimental parameters}

We investigated the growth of silver whiskers upon variations of mechanical pressure towards the cathode, deposition time and electric current. Longer deposition times at constant mechanical pressure result in longer whiskers without changing the diameter during growth. The practical limit to whisker length depends simply on the mechanical forces which easily lead to the deformation of long whiskers. During growth the electrode is detached and is continuously separated from the electrolyte by the growing whiskers, and a homogeneous mechanical pressure to the electrode is highly recommended. Typically, we grew whiskers with a diameter between a few microns and some ten microns and with a length of a few millimeters. The average whisker density has been determined as $2 \times 10^{6} / \mathrm{cm}^{2}$. If the electric current is reduced, the growth rate decreases, too. Faster growing whiskers show a larger diameter than others and are often grown together, leading to twins. Whiskers which are grown as twins can also be produced by applying higher mechanical pressure to the cell.

\section{Growth kinetics}

We observed the growth process in situ under an optical microscope (see Fig. 2) with a long distance optics. A typical slip band of a single silver whisker is marked with a white circle. As is seen in the time series, this mark moves along with the growing whisker during the experiment. We measured the length of a growing whisker as function of time and got a constant growth rate (cf. Fig. 3) for constant electric current. At a current of $I=150 \mu \mathrm{A}$ we measured a mean growth rate of $2.7 \mu \mathrm{m} / \mathrm{min}$ for a whisker with an approximate diameter of $1 \mu \mathrm{m}$, from which we evaluate a current density of $133 \mathrm{~mA} / \mathrm{cm}^{2}$ for the observed whisker.

\section{I-U-characteristics}

Applying low voltages in the order of some millivolts to the cell leads to currents in the order of up to $1 \mathrm{~mA}$. A typical cyclic voltammogram is shown in Fig. 4. The cathode always shows a completely reversible and ohmic behaviour corresponding to an exchange current of $I_{0} \cong$ $50 \mathrm{~mA} / \mathrm{cm}^{2}$, estimating the whisker root area to be approximately $8 \%$ of the geometrical area of the cell in this experiment. Thus, combining results and current density estimation for single whiskers and whisker ensembles, we conclude that a typical mean current density at $I=150 \mu$ A leads to local current densities at each whisker root in the order of $100 \mathrm{~mA} / \mathrm{cm}^{2}$. This

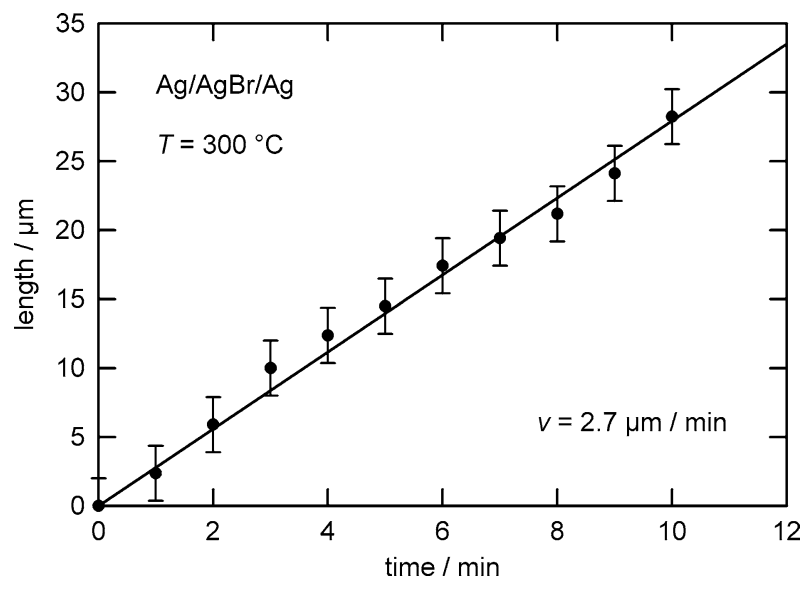

Fig. 3 Length of a silver whisker (marked in the left micrograph, galvanostatic conditions) as function of time
Fig. 2 Time sequence of optical micrographs taken during cathodic growth of a whisker array. One whisker with a diameter of approximately $1 \mu \mathrm{m}$ is marked. The outward shift of a whisker defect during growth clearly proves growth by metal addition at the $\mathrm{AgBr} /$ Ag-whisker interface $(I=$ $150 \mu \mathrm{A}, T=300{ }^{\circ} \mathrm{C}$ )
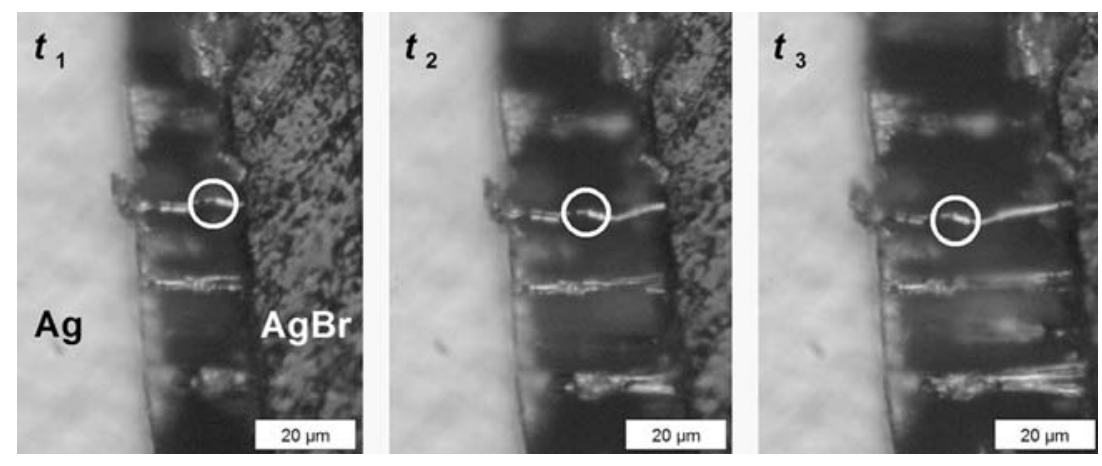


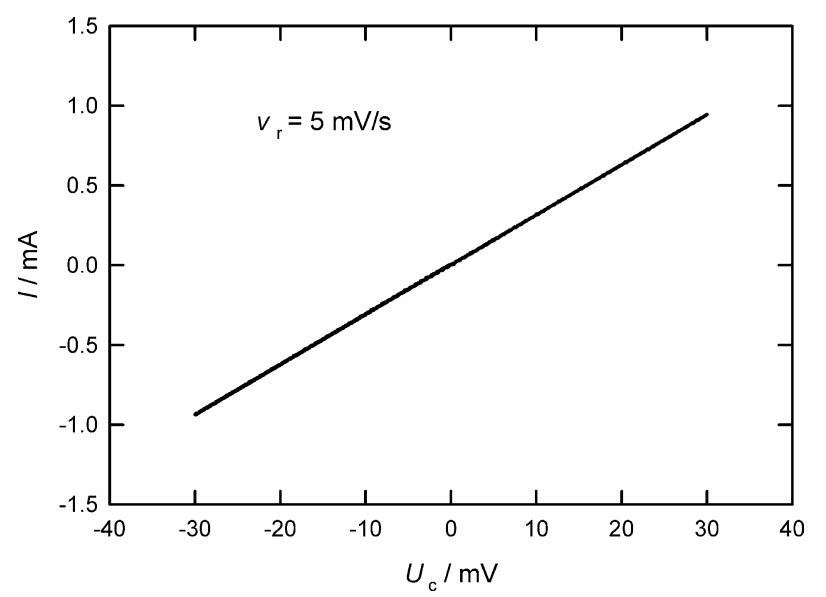

Fig. 4 Cyclic voltammogram of the cell $\mathrm{Ag} / \mathrm{AgBr} / \mathrm{Ag}$ during whisker growth and dissolution

value corresponds well with the observed whisker growth rate.

The current was applied to a cell with a geometrical electrode area of $0.25 \mathrm{~cm}^{2}$, but the electrochemically active area (the whisker/electrolyte interface area) results

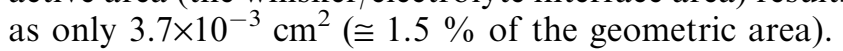

\section{Whisker roots}

The whisker roots have been investigated by scanning electron microscope (SEM) after cathodic growth. Typically, we found three different types of whisker roots (see Fig. 5a-c) after removal of the substrate, all showing a pyramidal shape with either fourfold, sixfold or spherical symmetry even for the same substrate $(\mathrm{AgBr}$ single crystal, not orientated). All whisker roots are grown into the solid electrolyte. Whether this fact has to be explained by plastic deformation of the relatively soft $\mathrm{AgBr}$ or is directly related to the nucleation process cannot be clarified yet. Most probably the whisker nucleation takes place at points where dislocations emerge at the surface of the solid electrolyte. These points are sites of repeatable growth and favour the nucleation process.

\section{Discussion}

Silver bromide is a good silver ion conductor with negligible electronic conductivity at $T=300{ }^{\circ} \mathrm{C}$. Thus the current is transformed 1:1 into a corresponding silver ion flux across $\mathrm{AgBr}$. Silver dissolution takes place at the anode and leads to the same non-linear kinetics as described by Majoni and Janek [8, 9] for the electrochemical cell $\mathrm{Ag} / \alpha-\mathrm{AgI} / \mathrm{Ag}$. At solid/solid electrodes the rigidity of both the crystalline electrolyte and the metal electrode and the corresponding mechanical properties usually lead to complicated anode kinetics even including periodic oscillatory behaviour. Origin of this complicated kinetics is the formation of metal vacancies and the relaxation of these lattice vacancies at the anodic interface. At low temperature these lattice vacancies create pores and lead to a more or less complete degradation of the electrode interface under continuous anodic load. At high temperatures the annihilation rate of vacancies increases due to an increase in atomic mobility. In any case, the anodic electrode process is extremely sensitive to mechanical pressure, and it is difficult to obtain reproducible experimental results.

As it is shown, the specific properties of solid/solid interfaces lead to cathodic growth modes which differ completely from their counterparts in liquid state. The cathodic deposition of silver on silver bromide occurs via reproducible formation of crystalline silver microwires
Fig. 5 SEM pictures (a-d). Individual whisker roots, a pyramidal, b cone-shaped, c stare-shaped, $\mathbf{d}$ hexagonal whisker
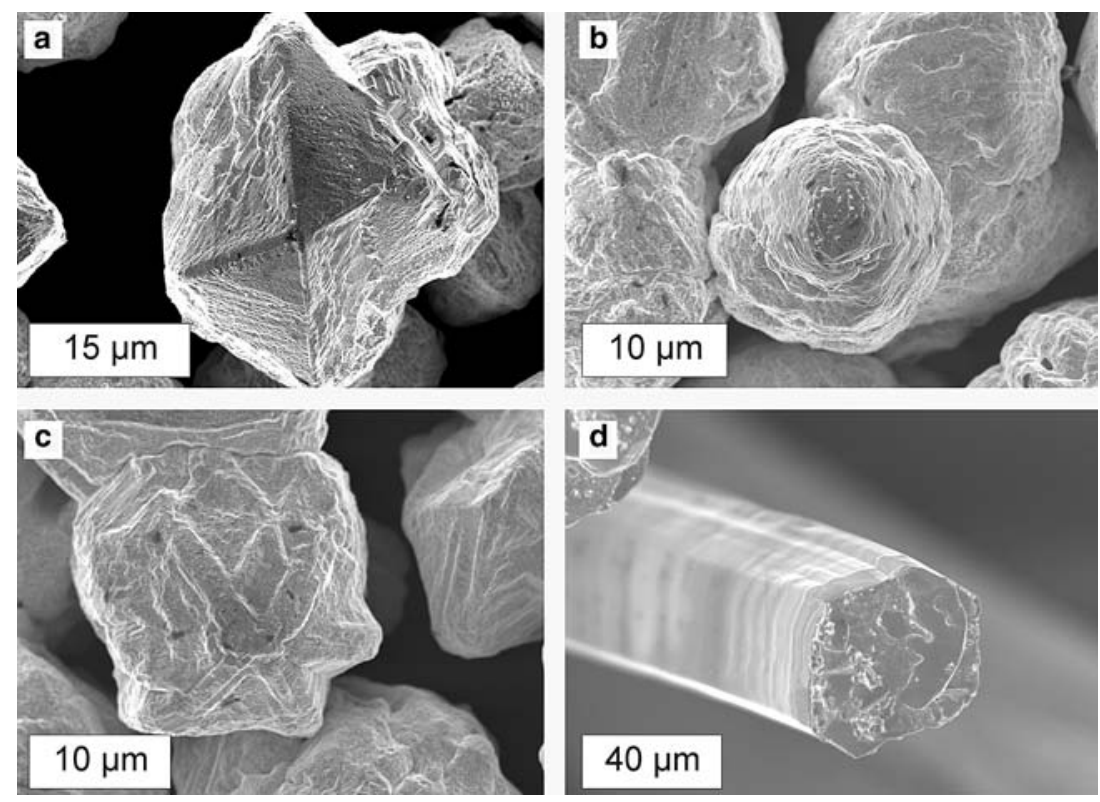
(whiskers) with typical diameters in the range from 1 to $20 \mu \mathrm{m}$ on large electrolyte areas in the order of many square millimeter with a density in the order of $10^{6} / \mathrm{cm}^{2}$.

It can be seen clearly in the time series of whisker growth in Fig. 2 that the marked slip band shifts outward. This proves that the whisker growth takes place at the interface $\mathrm{AgBr} / \mathrm{Ag}$-whisker (the "whisker root"). Thus, two other whisker growth mechanisms, the vapour-liquid-solid [9] and the spiral growth mechanism [10] which are described in the literature for other systems, can definitely be excluded. Whisker growth on $\mathrm{Ag}_{2} \mathrm{~S}$ driven by supersaturation of $\mathrm{Ag}$ has been investigated by O'Briain [3] and Ohachi [4]. Both also proved that whisker growth occurred by metal addition to the whisker root. Again, the morphology of the whiskers is different.

The excellent electrode properties of whiskers easily allow high current densities, as only a few percent of the geometrical area of a substrate are in contact. Since the whiskers have a more or less higher distance between each other, the real electrochemically active contact area is significantly smaller than the geometrical area. The observed current exchange density in the order of approximately $100 \mathrm{~mA} / \mathrm{cm}^{2}$ is not as high as the one determined by Corish and $\mathrm{O}^{\prime}$ Briain at a single Ag-whisker in contact with $\mathrm{Ag}_{2} \mathrm{~S}$. They reported a current exchange density of up to $2 \mathrm{~A} / \mathrm{cm}^{2}$.

It is striking that silver whiskers obviously show, at least partially, a hcp structure. Regarding the small difference in lattice energy for silver in its equilibrium fcc and metastable hep structure, this is not too surprising. We suppose that the electrochemical growth occurs at a sufficiently strong deviation from thermodynamic equilibrium, such that non-equilibrium structures are created despite the elevated temperature. Recently, Jona et al. [13] made total-energy calculations for hexagonal silver and showed that hexagonal silver represents a metastable phase. Such kind of strong disorder is not impossible. For example, it is also well known that cobalt metal shows a high degree of stacking disorder [14]. Interestingly a hexagonal fcc lattice was found for nano-crystalline super silver particles by Harfenist et al. [12].

\section{Conclusions}

Summarizing, we find that cathodic deposition of silver on $\mathrm{AgBr}$ at extended electrodes at a temperature of $T=$ $300{ }^{\circ} \mathrm{C}$ leads rather to the growth of whisker arrays or fields than to fractal or dendritic surface morphologies as reported by Spangenberg et al. [1] for $\mathrm{AgCl}$. Whether this is due to a different surface chemistry of $\mathrm{AgCl}$ and $\mathrm{AgBr}$ is yet not clear. We assume that the size of the cathode and the electric field distribution influence the growth mode. Dendritic or fractal growth is usually observed at point electrodes, whereas we observe whisker growth only at electrodes with a large electroactive area.
We assume that surface defects in the solid electrolyte formed by the points of emergence of dislocations create sites for the nucleation of whiskers. However, so far this is a hypothesis rather than a proven fact. A typical whisker density is in the order of $2 \times 10^{6} / \mathrm{cm}^{2}$ which compares relatively well with typical dislocation densities in ionic crystals and supports our hypothesis. Whether regular whisker arrays can be produced after a suitable surface preparation (e.g. electron beam lithography) will be investigated by further experiments. In addition, we examine whether other metals can be deposited on adequate solid electrolyte substrates (e.g. copper whiskers on copper halides). An important aspect to be investigated is the strong influence of the substrate on the product morphology.

Finally, it has to be noted that Ohachi [15] reported on the electrochemical growth of silver whiskers on silver sulphide and silver selenide. The growth mechanism is different, as Ohachi produced whiskers by supersaturation of the non-stoichiometric material. Thus, the position and density of whiskers is not controlled via an electrode process, as it is shown in our work. The cathodic deposition of silver on single crystalline $\mathrm{AgCl}$ has been investigated by Spangenberg et al. [1] employing a microelectrode technique. The growth occurs mainly along mechanically produced surface defects like scratches. These are decorated by the cathodic deposit, and thus, electrochemical "writing" on a solid electrolyte surface is possible. The solid state electrochemical growth of silver or copper microwires, presented here, might easily be improved by using prestructured electrolytes.

Acknowledgements We are grateful to A. Dülmer and Dr. W. Herrendorf (Institut für Anorganische und Analytische Chemie, Justus-Liebig-Universität Gießen) for performing XRD measurements. The study is part of a project funded by the DFG (DFG Ja 648/6-1).

\section{References}

1. Spangenberg A, Fleig J, Maier J (2001) Adv Mater 13:1466

2. Terabe K, Nakayama T, Hasegawa T, Aono M (2002) J Appl Phys 91:10110

3. Corish J, O'Brian CD (1972) J Cryst Growth 13(14):62

4. Ohachi T, Taniguchi I (1972) J Cryst Growth 13(14):191

5. Schmalzried H, Wagner C (1963)Trans Met Soc 227:539

6. Budevski EB, Staikov GT, Lorenz WJ (1998) Electrochemical phase formation and growth. Wiley-VCH Verlag, Weinheim

7. Haasen P, Haasen P (1996) Physical metallurgy. Cambridge University Press, Cambridge

8. Majoni S, Janek J (1995) Ber Bunsenges Phys Chem 99:14

9. Majoni S, Janek J (1998) Ber Bunsenges Phys Chem 102:756

10. Wagner RS, Ellis WC (1964) Appl Phys Lett 4:89

11. Frank FC (1953) Phil Mag 2:857

12. Harfenist SA, Wang ZL, Whetten RL, Vezmar I, Alvarez MM (1997) Adv Mat 9:817

13. Jona F, Marcus PM (2004) J Phys Condens Matter 16:5199

14. Kajiwara S (1970) Jap J Appl Phys 9:385

15. Ohachi T (1974) Solid state ionics of silver compounds. PhD Thesis, Doshisha University 